\title{
Maximizing the Capture of the Excitable Gap During Ventricular Arrhythmias for Low-Energy Defibrillation
}

\author{
Angel Moreno $^{1,2}$, Richard D Walton ${ }^{1,3}$, Olivier Bernus ${ }^{1,3}$, Edward J Vigmond ${ }^{1,2}$, Jason D Bayer ${ }^{1,2}$ \\ ${ }^{1}$ Electrophysiology and Heart Modeling Institute (IHU LIRYC), Bordeaux University Foundation, \\ Bordeaux, France \\ ${ }^{2}$ Institute of Mathematics of Bordeaux (UMR 5251), University of Bordeaux, Bordeaux, France \\ ${ }^{3}$ Cardio-Thoracic Research Center of Bordeaux (INSERM U1045), University of Bordeaux, \\ Bordeaux, France
}

\begin{abstract}
Aim: To optimize the capture of the excitable gap for low-energy defibrillation with line electrodes.

Methods: A finite element model of left ventricular $(L V)$ tissue with human electrophysiology parameters was used to study ventricular arrhythmias and defibrillation. At the beginning of the simulation, 1 second of steady-state was applied to load the parameters, followed by 10, $2 \mathrm{~ms}$ long $S 1$ pulses at $1.13 \mathrm{~Hz}$ (apex - base propagation). The LV model was then preconditioned with $S 2$ pulses at $2.5 \mathrm{~Hz}$ from a line located in the middle of the model. To induce reentry, the tissue was paced with $10 \mathrm{~S} 3$ pulses at $4.25 \mathrm{~Hz}$ from the same line. To defibrillate, a single, S4 pulse was delivered with coupling intervals incremented by $25 \mathrm{~ms}$ from multiple lines equally spaced in an apical-basal orientation across the tissue.

Results: A line electrode spacing of $0.25 \mathrm{~cm}$ terminated reentry regardless of timing and under $100 \mathrm{~ms}$ after the $S 4$ stimulus, while capturing over $40 \%$ of the excitable gap. The most optimal timing to deliver the defibrillation stimulus is between 25-50 ms after depolarization.

Conclusion: Proper electrode placing and timing was necessary to capture $>40 \%$ of the excitable gap, which was necessary to consistently terminate ventricular arrhythmias.
\end{abstract}

\section{Introduction}

\subsection{Background}

The extremely disorganized electrical activity in the ventricles during ventricular fibrillation (VF) is one of the leading causes of sudden cardiac death [1]. Given the imminent loss of cardiac output during a VF episode, prompt care is required to avoid complete organ failure.

Typically, a strong far-field shock is administered to terminate VF by rapidly depolarizing the entire cardiac tissue. Despite being the gold standard for defibrillation, several adverse effects such as myocardial damage, pain and anxiety disorders [2]-[4], have been observed in patients with implantable cardiac defibrillators (ICD) after the delivery of high-energy shocks.

To mitigate these risks, several low-energy defibrillation alternatives via surface stimulation have been proposed, including the targeting of the excitable gap (EG) by activating genetically modified cardiac cells to block reentrant circuits [5]. However, the effectiveness of this technique relies on the location and duration of the EG concerning the pacing sites, which for VF could be unpredictable. Likewise, the translation of this approach to large mammalian ventricles for defibrillation is challenging, particularly regarding electrode placing and timing.

\subsection{Aims}

This investigation aims to determine the amount of capture of the EG with respect to the location and timing of the defibrillation stimuli.

\section{Methods}

\subsection{LV wedge model}

A large $10 \mathrm{~cm} \times 7 \mathrm{~cm} \times 475 \mu \mathrm{m}$ model of LV tissue was used to simulate and study ventricular arrhythmia and defibrillation. The size of the model was chosen to be $>2 \mathrm{x}$ the wavelength for reentry to allow for long lasting $(>5 \mathrm{~s})$ ventricular arrhythmias. The volume was discretized at $475 \mu \mathrm{m}$ with 31017 elements and 62752 nodes. Fiber orientation in the model was uniform and parallel to the apico-basal axis. Conduction velocity in the model was adjusted to match previous studies [6]. Membrane kinetics at the cellular level were described with a ten Tusscher- 
Panfilov model [7] with modification of the potassium currents along the apico-basal direction to generate a repolarization gradient with APD >100 ms longer the base than apex of the model.

Simulations were performed with the Cardiac Arrhythmia Research Package (Cardiosolv, LLC) and were monodomain using a time step of $20 \mu \mathrm{s}$ on four compute nodes each with two Hexa-Core Intel Xeon X5675 @ 3.06 Ghz CPUs and 48 GB of memory.

\subsection{VF initiation protocol}

1 second of simulation without any stimulation was performed before the pacing protocols to allow the model to load and equilibrate all the parameters. Subsequently, the LV tissue model was paced for 10 beats at a cycle length (CL) of $750 \mathrm{~ms}$, with a $2 \mathrm{~ms}$ long S1 transmembrane current stimuli delivered at twice capture threshold $(2 \mathrm{x}$ thr.) along the bottom apical edge of the model. The LV model was then preconditioned during 10 beats with a $2 \mathrm{~ms}$ long, 2x thr. S2 stimuli pacing at a CL of $400 \mathrm{~ms}$ from a 1 $\mathrm{mm}$ (diameter) single line located in the middle of the LV, and with an apico-basal orientation. Next, reentrant arrhythmia resembling VF was induced with 10, S3 stimuli at a CL of $235 \mathrm{~ms}$ from the same line (Figure 1). This protocol matches an animal protocol being validated in porcine hearts to generate reentry, with tissue properties and ventricular dimensions close to humans.

\subsection{Low-energy defibrillation}

To terminate the arrhythmia, a single low-energy $2 \mathrm{~ms}$ long S4 stimulus was applied uniformly from lines evenly spaced $0.25-2 \mathrm{~cm}$ apart across the tissue with an apicobasal orientation (Figure 1). To determine how reentry termination depends on stimulus timing, defibrillation was attempted with varying delay $(25 \mathrm{~ms}$ increment from 0 to $5 \mathrm{sec}$ after VF initiation) from the end of the VF induction protocol. To ensure homogeneous activation across the lines and a uniform planar wave front propagation, the stimulus threshold was established at $8 \mathrm{x}$ threshold determined by large animal studies [8]. When defibrillating, uniform stimulation with line electrodes increases the possibility of blocking reentrant activation fronts.

\subsection{Data analysis}

Arrhythmia complexity was determined every $250 \mathrm{~ms}$ and calculated by analyzing the dominant frequency. The excitable gap was established as the percentage of tissue with membrane potential <-70 $\mathrm{mV}$. Reentry was systematically considered terminated if membrane potential returned to rest after $1 \mathrm{sec}$ of simulation. Data were presented as mean \pm standard error of the mean.

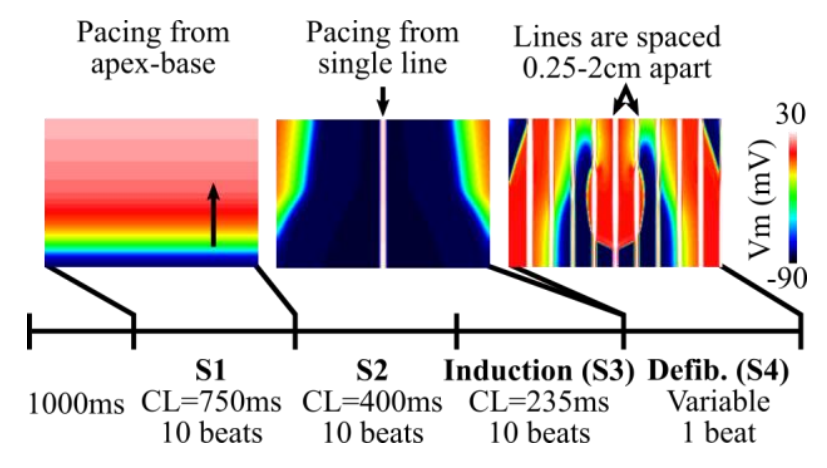

Figure 1. Protocol for tissue preconditioning, VF induction and defibrillation via surface stimulation.

\section{Results}

\subsection{VF initiation and defibrillation}

VF resulted from reentry in the LV wedge model with a dominant frequency of $4.17 \mathrm{~Hz}$ and an average cycle length of $240 \mathrm{~ms}$. Two stable rotors, one at each side of the induction line electrode forming a figure-of-eight reentrant pattern, were observed throughout the 5 secs of simulation post S3 pacing for VF initiation (Figure 2A). Importantly, in some cases, reentry complexity increased over time if the $\mathrm{S} 4$ defibrillation pulse was unsuccessful.

As a control, low-energy defibrillation was successful when stimulating all the nodes located within the excitable gap (Figure 2B), regardless of the time interval. VF was instantly terminated after the S4 pulse.
A

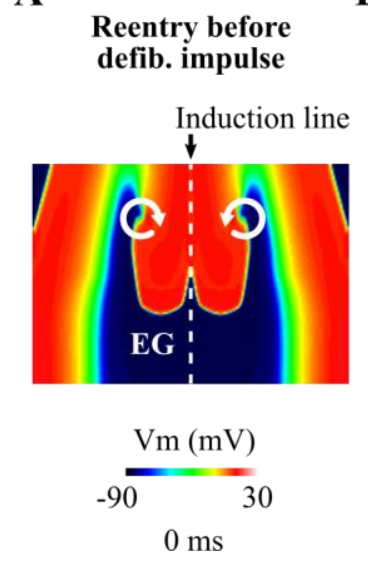

B

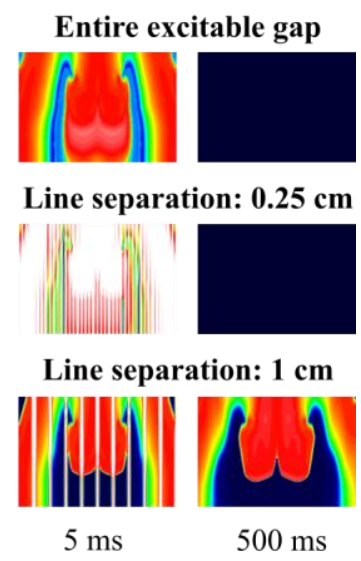

Figure 2. VF initiation and termination. A. Figure-of-eight reentry induced via rapid pacing from a single line electrode. B. Stimulation of the entire EG or via lines electrodes separated every $0.25 \mathrm{~cm}$ rapidly terminated $\mathrm{VF}$, whereas lines electrode spaced farther apart at $1 \mathrm{~cm}$ had a diminished success rate.

A linear relationship was observed when attempting to 
terminate reentry via line stimulation (Table 1). Line electrodes placed every $0.25 \mathrm{~cm}$ yielded the highest efficiency by defibrillating the LV tissue model for $100 \%$ of the S4 stimuli over the $5 \mathrm{sec}$ time period post VF initiation. In this case, VF termination was accomplished under $100 \mathrm{~ms}$ from the stimulus application and when the line electrodes occupied on average $42 \%$ of the EG.

Defibrillation success declined from $56 \%$ to $30 \%$ of the S4 over 5 secs post VF initiation when having a line separation from 0.5 to $2 \mathrm{~cm}$ respectively. Likewise, the mechanism of termination varied in certain instances. Out of all cases where reentrant termination was possible, 10 to $30 \%$ were considered as having a delayed defibrillation, where reentrant activity was sustained for over $500 \mathrm{~ms}$ (after stimulus) but less than $1200 \mathrm{~ms}$ before selftermination by colliding wavefronts, as opposed to when the reentrant circuits were rapidly blocked after S4 during the entire stimulation of the EG and the lines at $0.25 \mathrm{~cm}$.

Table 1. Overall performance of S4 defibrillating pulses with respect to line electrode spacing.

\begin{tabular}{cccc}
\hline \hline $\begin{array}{c}\text { Line } \\
(\mathbf{c m})\end{array}$ & $\begin{array}{c}\text { Success } \\
(\boldsymbol{\%})\end{array}$ & $\begin{array}{c}\text { Time until VF } \\
\text { termination }(\mathbf{m s})\end{array}$ & $\begin{array}{c}\text { Line nodes } \\
\text { in EG }(\boldsymbol{\%})\end{array}$ \\
\hline 0.25 & 100 & $72 \pm 4$ & $42 \pm 0.3$ \\
0.5 & 56 & $381 \pm 32$ & $18 \pm 0.1$ \\
1 & 38 & $455 \pm 55$ & $10 \pm 0.3$ \\
2 & 30 & $525 \pm 65$ & $5 \pm 0.5$ \\
\hline \hline
\end{tabular}

Lastly, optimal timing for the defibrillation stimulus was determined between 25-50 ms after the depolarization of a node adjacent to a phase singularity (Figure 3 ). Within this period, termination is possible in about $83 \%$ of the S4 compared to $53 \%$ during a different timeframe, by effectively blocking the path of the rotor.

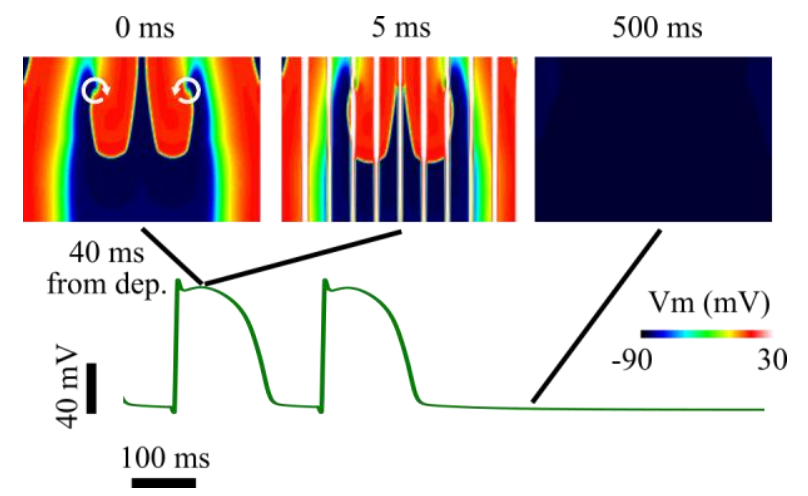

Figure 3. Line electrode stimulation spaced $1 \mathrm{~cm}$ apart terminated reentry when delivered $45 \mathrm{~ms}$ after depolarization.

\section{Conclusions}

Low-energy defibrillation is achievable via a single defibrillation pulse delivered by line electrodes spaced $\leq 2$ $\mathrm{cm}$ across the ventricular surface. Line electrodes capture the excitable gap to block reentrant circuits, thus restoring sinus rhythm after VF. Since capturing the entire excitable gap in practice is challenging with surface stimulation given the unpredictable nature of reentrant circuits, this study provides evidence that it is feasible to terminate VF with only partial capture of the excitable gap $(<42 \%)$. Furthermore, our research suggests that the success rate for defibrillation could improve with the proper timing of the stimulus to capture the excitable gap at just the right moment.

Future studies will investigate the effects of tissue heterogeneities and conduction gradients on arrhythmia complexity and defibrillation success in a more complex ventricular model, as well as, determine the optimal location and shape of the electrodes to maximize the capture of excitable tissue.

Finally, the studying is currently being repeated using different arrhythmias states to assess low-energy defibrillation via surface stimulation in the presence of one or more rotors at different frequencies. This will help to determine the performance in a more real clinical scenario where arrhythmia complexity changes over time.

\section{Acknowledgments}

This research was funded by the French National Research Agency grants ANR-10-IAHU-04 and ANR-16CE19-0009.

\section{References}

[1] M. Hayashi, W. Shimizu, and C. M. Albert, "The Spectrum of Epidemiology Underlying Sudden Cardiac Death," Circ. Res., vol. 116, no. 12, pp. 1887-1906, 2015.

[2] J. Brewster et al., "Acute Effects of Implantable Cardioverter-Defibrillator Shocks on Biomarkers of Myocardial Injury, Apoptosis, Heart Failure, and Systemic Inflammation," PACE - Pacing Clin. Electrophysiol., vol. 40, no. 4, pp. 344-352, 2017.

[3] D. M. Steinhaus, D. S. Cardinal, L. Mongeon, S. K. Musley, L. Foley, and S. Corrigan, "Internal Defibrillation: Pain Perception of Low Energy Shocks," PACE - Pacing Clin. Electrophysiol., vol. 25, no. 7, pp. 1090-1093, 2002.

[4] G. Magyar-Russell et al., "The Prevalence of Anxiety and Depression in Adults With Implantable Cardioverter Defibrillators: A Systematic Review," J. Psychosom. Res., vol. 71, no. 4, pp. 223-231, 2011.

[5] P. Sasse, M. Funken, T. Beiert, and T. Bruegmann, "Optogenetic Termination of Cardiac Arrhythmia: Mechanistic Enlightenment and Therapeutic Application?," Front. Physiol., vol. 10, no. JUN, 2019.

[6] J. Bayer, G. Lalani, E. Vigmond, S. Narayan, and N. Trayanova, "Mechanisms Linking Electrical Alternans and Clinical Ventricular Arrhythmia in Human Heart Failure," Hear. Rhythm, vol. 13, no. 9, pp. 1922-1931, 2016.

[7] K. H. W. J. Ten Tusscher and A. V. Panfilov, "Alternans and 
Spiral Breakup in a Human Ventricular Tissue Model," Am. J. Physiol. - Hear. Circ. Physiol., vol. 291, no. 3, pp. 10881100, 2006.

[8] A. Moreno, R. D. Walton, M. Constantin, O. Bernus, E. J. Vigmond, and J. D. Bayer, "Wide-Area Low-Energy Surface Stimulation of Large Mammalian Ventricular Tissue," Sci. Rep., vol. 9, no. 1, p. 15863, 2019.

Address for correspondence:

Jason D. Bayer

IHU LIRYC - Campus Xavier Arnozan

Avenue du Haut Leveque

33600 Pessac

France

jason.bayer@ihu-liryc.fr 